\title{
Model based Controller Design for a Spherical Tank
}

\author{
M.Bharathi, C.Selvakumar, A.Kalpana \\ HOD, Department of Electronics and Instrumentation Bharath University, Chennai-73 \\ Professor \& Head, St.Joseph's College Of Engineering, Chennai-119 \\ PG Student, EIE, Bharath University, Chennai-73
}

\begin{abstract}
The aim of this paper is to implement optimum controller for a spherical tank. The objective of the controller is to maintain the level inside the process tank in a desired value. The real time implementation of the process is designed and implemented in MATLAB using data acquisition module. The identified model is in the form of first order plus delay time (FOPDT) process. Controller design is compared based on conventional Proportional Integral Derivative (PID) with Internal Model Control (IMC) based on Skogestad's settings in terms of performance indices. Out of the control algorithms SIMC outperforms in no overshoot, faster settling time, better set point tracking and produces lower performance indices.
\end{abstract}

Key words: Process modeling, Data acquisition system, PID controller, Internal Model Controller (IMC), FOPDT, SIMC, Spherical tank

\section{Introduction}

Chemical process present many challenging control problems due to nonlinear dynamic behavior, uncertain and time varying parameters, constraints on manipulated variable, interaction between manipulated and controlled variables, unmeasured and frequent disturbances, dead time on input and measurements. Because of the inherent nonlinearity, most of the chemical process industries are in need of conventional control techniques. Spherical tanks find wide spread usage in gas plants. They are non-linear system because their area of cross-section keeps varying with the height of the tank. A sphere is a very strong structure. The even distribution of stresses on the sphere's surfaces means that there are no weak points. Moreover, they have a smaller surface area per unit volume than any other shape of vessel. This means, that the quantity of heat transferred from warmer surroundings to the liquid in the sphere, will be less than that for cylindrical or rectangular storage vessels. Thus causing less pressurization due to external heat. Control of a spherical tank is important, because the change in shape gives rise to the nonlinearity. The proportional-integral (PI) and proportional-integral-derivative (PID) control modes are used in many industries.

In this paper, we propose real time SIMC a model based controller for controlling the liquid level in a spherical tank. The basis for SIMC method is a first order plus time delay model. SIMC is a modified form of Internal Model Control, where the integral term is modified to improve disturbance rejection for integrating process. Single tuning rule is used for both first order and second order plus time delay model rather than separate rules for each model. SIMC performances are compared with conventional controller based on integral square error (ISE) and integral absolute error (IAE) for set point and load disturbances.

The most basic and pervasive control algorithm used in the feedback control is the Proportional Integral Derivative (PID) algorithm. PID control is widely used control strategy to control most of the industrial automation process because of the industrial automation process because of its remarkable efficiency, simplicity of approximated by a first order time delayed model and suggested frequency domain approach based on normalized open loop transfer function to evaluate the effects of uncertainties in the process parameters and thus control system robustness.

\section{Block Diagram}

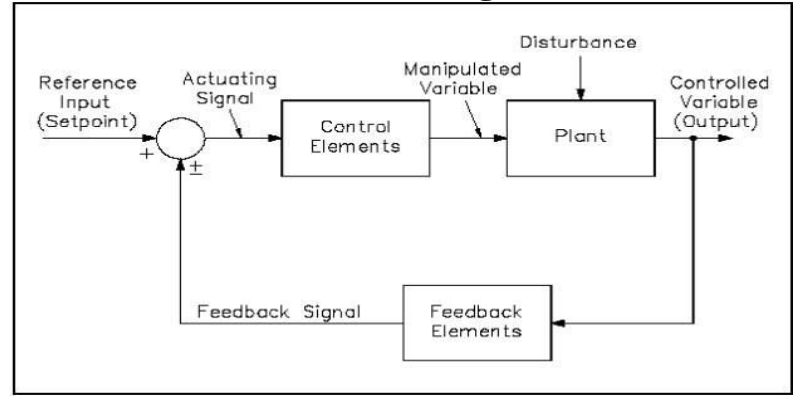

Figure - 1: Block Diagram 


\section{Mathematical Modeling Of The Spherical Tank System}

Any system, if has to be analyzed, must be mathematically modeled, i.e. the mathematical equations describing the system must be derived so as to aid in studying the system, its features, predicting the dynamic behavior \& for many other purposes. The mathematical modeling the system is as follows:

Consider a spherical tank, as shown in figure 4 , of radius $\mathrm{R}$. The water flows in at a rate $\mathrm{F}_{\text {in }} \&$ flows out at a rate $\mathrm{F}_{\text {out }}$.

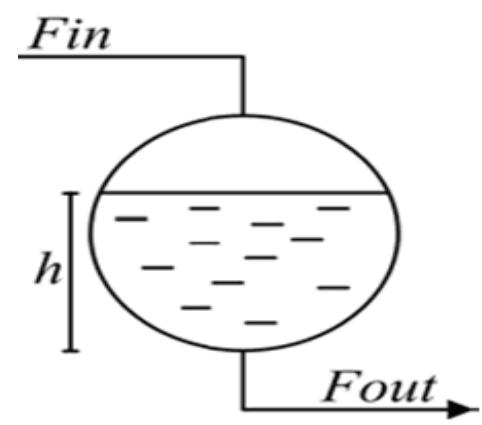

Volume of a sphere is given by, $V=\frac{4}{3} \pi h^{3}$

Figure - 2: Mathematical model of a spherical tank

The first order differential equation of the system is given by,

$F_{i}-$ Flow rate at inlet of the tank

$F_{o}-$ Flow rate at outlet of the tank

$h$ - Height of the liquid in the tank

$R$-Resistance to flow

$\mathrm{F}_{\mathrm{o}}=\frac{\mathrm{h}}{\mathrm{R}}$

A-Area of cross-sectional area of tank

$$
A \frac{d h}{d t}=F_{i}-F_{o}=F_{i}-\frac{h}{R}
$$

$\mathrm{AR} \frac{\mathrm{dh}}{\mathrm{dt}}+\mathrm{h}=\mathrm{RF}_{\mathrm{i}} \quad \rightarrow(1)$

At steady state

$\mathrm{h}_{\mathrm{s}}=\mathrm{RF}_{\mathrm{i}, \mathrm{s}}$

In terms of deviation variables from (1) and (2)

$\mathrm{AR} \frac{\mathrm{dh}^{\prime}}{\mathrm{dt}}+\mathrm{h}^{\prime}=\mathrm{RF}_{\mathrm{i}}{ }_{\mathrm{i}}$

Where $\mathrm{h}^{\prime}=\mathrm{h}-\mathrm{h}_{\mathrm{s}}$ and $\mathrm{F}_{\mathrm{i}}^{\prime}=\mathrm{F}_{\mathrm{i}}-\mathrm{F}_{\mathrm{ts}}$

$\tau_{p}=A R=$ time constant of the process

$K_{p}=R=$ steadystate gain of the process

Transfer function is

$\mathrm{G}(\mathrm{s})=\frac{\mathrm{h}^{\prime}(\mathrm{s})}{\mathrm{F}^{\prime}{ }_{\mathrm{i}}(\mathrm{s})}=\frac{\mathrm{K}_{\mathrm{p}}}{\tau_{\mathrm{n}} \mathrm{s}+1}$

$\mathrm{G}(\mathrm{s})=\frac{\mathrm{H}(\mathrm{s})}{\mathrm{Q}(\mathrm{s})}=\frac{\mathrm{R}_{\mathrm{t}}}{\tau \mathrm{s}+1}$

$\mathrm{R}_{\mathrm{t}}=\frac{2 \mathrm{~h}_{\mathrm{s}}}{\mathrm{q}_{2}}$

Where $h_{s}=$ Height of the tank at the steady state

$\tau=4 \pi h_{s} R_{t}$

Time Constant $=$ Storage Capacity $\times$ Resistance to flow

\section{Design Of Pid Controller}

After deriving transfer function model the controller has to be designed for maintaining the system to the optimal set point. This can be achieved by properly selecting the tuning parameters Kp and for a PID controller. The tuning methods used in this paper are as follows: 


\section{ZEIGLER - NICHOLS (ZN) METHOD}

$\mathrm{K}_{\mathrm{p}}=\frac{\mathrm{K}_{\mathrm{u}}}{1.7}$

$\mathrm{T}_{\mathrm{i}}=\frac{\mathrm{P}_{\mathrm{u}}}{2}$

$\mathrm{T}_{\mathrm{d}}=\frac{\mathrm{P}_{\mathrm{u}}}{8}$

\section{COHEN - COON METHOD}

$\mathrm{K}_{\mathrm{p}}=\frac{1}{\mathrm{~K}} \cdot \frac{\tau}{\mathrm{t}_{\mathrm{d}}} \cdot\left[\frac{4}{3}+\frac{\mathrm{t}_{\mathrm{d}}}{4 \tau}\right]$

$T_{i}=t_{d}\left[\frac{32+6 \frac{t_{d}}{\tau}}{13+8 \frac{t_{d}}{\tau}}\right]$

$\mathrm{T}_{\mathrm{d}}=\mathrm{t}_{\mathrm{d}}\left[\frac{4}{11+2 \frac{\mathrm{t}_{\mathrm{d}}}{\tau}}\right]$

\section{IMC METHOD}

Internal model control is model based controller. The Figure 3 shows the IMC structure which makes use of a process model to infer the effect of immeasurable disturbance on the process output and then counteracts that effect. The controller consists of an inverse of the process model.

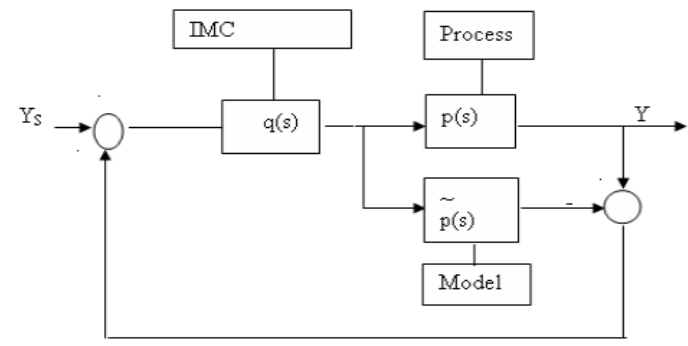

Figure - 3: IMC Structure

$\mathrm{p}(\mathrm{s})=\frac{\mathrm{kl}^{-\tau \mathrm{D}_{\mathrm{s}}}}{\tau \mathrm{s}+1}$

$q(s)=q(s)^{-} f$

$q(s)^{-}=\frac{\tau s+1}{K}$

From the above equation, the only tuning parameter is $\lambda$ and hence IMC controller is simple.

\section{SIMC METHOD}

Here we used a P-controller with a gain that is about half this value, such that the resulting overshoot (D) to a step change in the setpoint is about $30 \%$ (that is, $\mathrm{D}$ is about 0.3 ). We here describe the procedure proposed by Shamsuzzoha and Skogestad (2010) which seems to use the most easily available parameters from the closed-loop response. The system should be at steady-state initially, that is, before the setpoint change is applied. Then, from the closed-loop setpoint response one obtains the following parameters (see Figure 4).

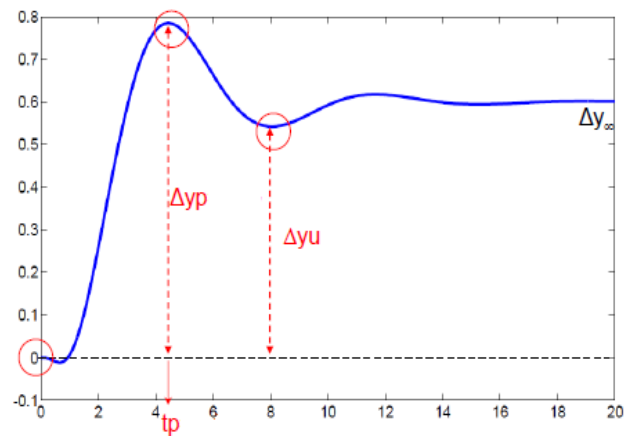

Figure - 4: Extracting information from closed-loop setpoint response with P-only controller. 
- Controller gain used in experiment, $\mathrm{K}_{\mathrm{c} 0}$

- Setpoint change, $\Delta y_{s}$

- Time from setpoint change to reach first (maximum) peak, $t_{p}$

- Corresponding maximum output change, $\Delta y_{p}$

- Output change at first undershoot, $\Delta y_{u}$

Steady-state output change (at infinite time) $\Delta \mathrm{y}_{\infty}=0.45\left(\Delta \mathrm{y}_{\mathrm{p}}+\Delta \mathrm{y}_{\mathrm{u}}\right)$

Overshoot $\mathrm{D}=\frac{\Delta \mathrm{y}_{\mathrm{p}}-\Delta \mathrm{y}_{\infty}}{\Delta \mathrm{y}_{\infty}}$

Steady-state offset $B=\left|\frac{\Delta y_{s}-\Delta y_{\infty}}{\Delta y_{\infty}}\right|$

We compute the parameters as below:

$\mathrm{A}=1.152 \mathrm{D}^{2}-1.607 \mathrm{D}+1$

$\mathrm{r}=2 \mathrm{~A} / \mathrm{B}$

$\mathrm{k}=1 /\left(\mathrm{K}_{\mathrm{c} 0} \mathrm{~B}\right)$

$\theta=\mathrm{t}_{\mathrm{p}} \cdot\left(0.309+0.209 \mathrm{e}^{-0.61 \mathrm{r}}\right)$

$\tau_{1}=r \theta$

For a first-order model

$g_{1}(s)=\frac{k}{\left(\tau_{1} s+1\right)} e^{-\theta s}$

The SIMC method results in a PI controller with settings

$\tau_{1}=\min \left\{\tau_{1}, 4\left(\tau_{c}+\theta\right)\right\}$

$$
\mathrm{K}_{\mathrm{c}}=\frac{1}{\mathrm{k}} \frac{\tau_{1}}{\tau_{\mathrm{c}}+\theta}=\frac{1}{\mathrm{k}^{\prime}} \frac{1}{\tau_{\mathrm{c}}+\theta}
$$

\section{Pid Settings Tuned By Various Methods}

$$
\mathrm{p}(\mathrm{s})=\frac{1.6}{5.026 s+1}
$$

\begin{tabular}{|l|r|r|r|}
\hline \multicolumn{1}{|c|}{ Controller } & \multicolumn{1}{c|}{$\mathbf{K}_{\mathbf{p}}$} & \multicolumn{1}{c|}{$\boldsymbol{\tau}_{\mathbf{1}}$} & \multicolumn{1}{c|}{$\boldsymbol{\tau}_{\mathbf{d}}$} \\
\hline Cohen Coon & 54 & 0.244 & 0.362 \\
\hline ZN & 2.196 & 0.9 & 0.225 \\
\hline SIMC & 4.5235 & 0.94 & 0 \\
\hline
\end{tabular}

Table - 1: Time domain comparison

Based on the recommended tuning parameters given in Table-1 the servo regulatory responses were obtained.

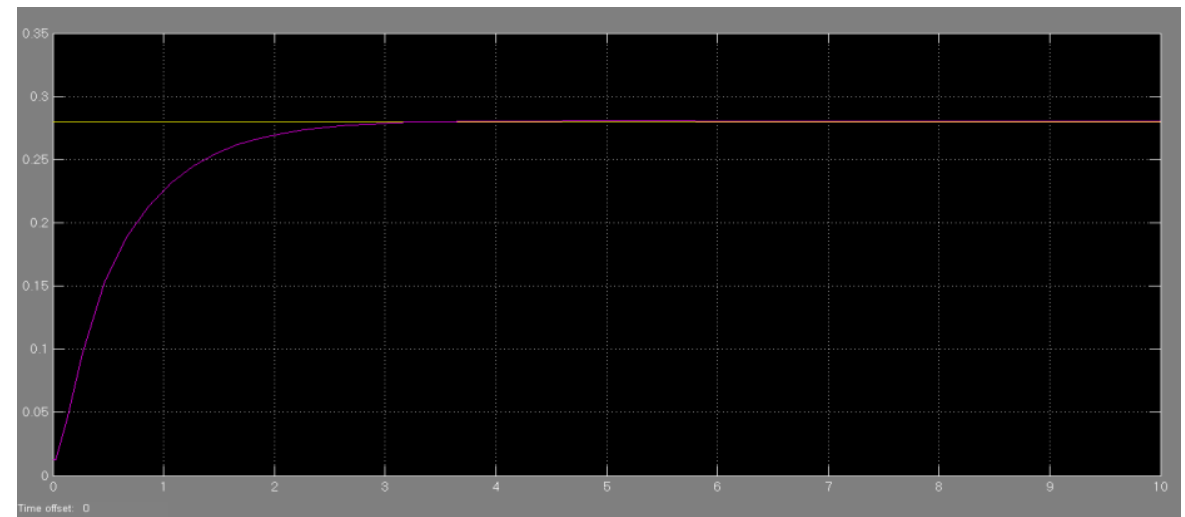

Figure - 5: Servo regulatory response of PI controller (SIMC) 


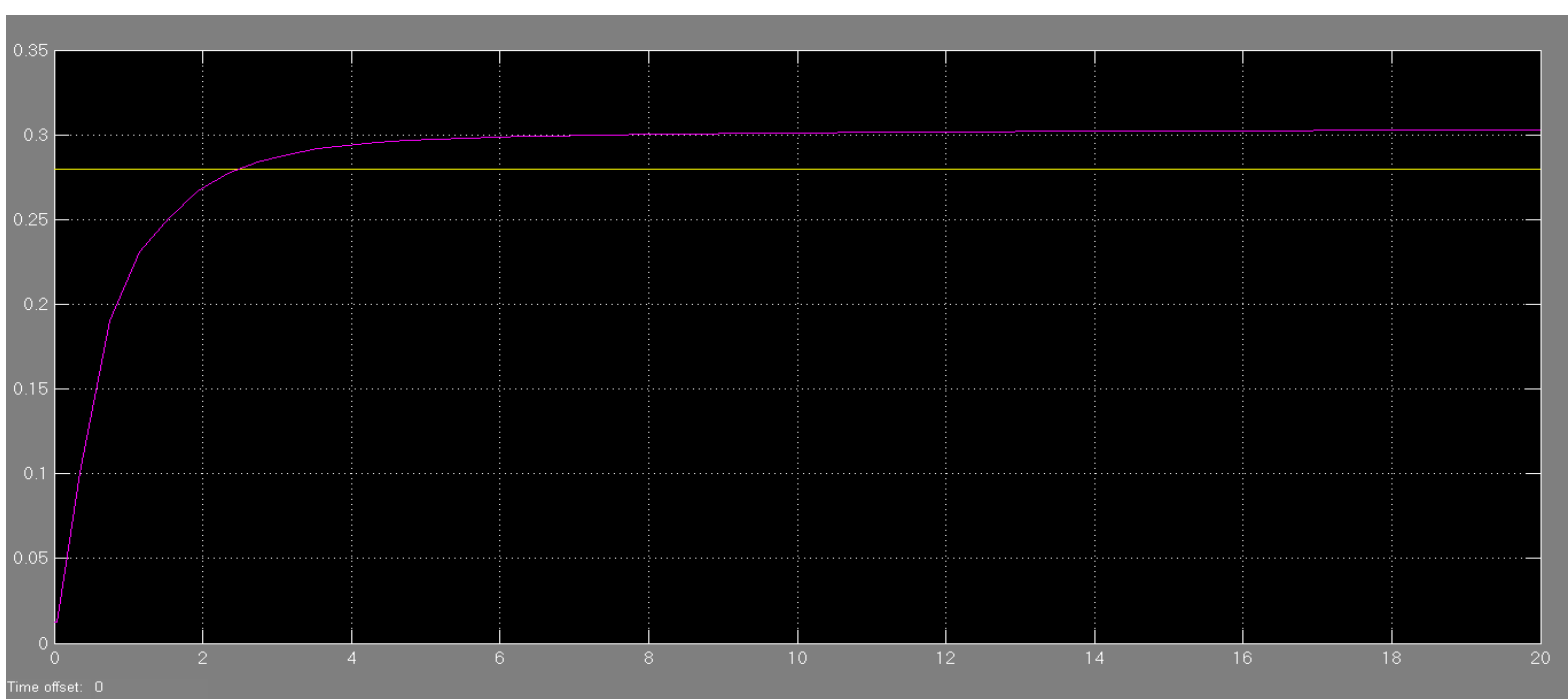

Figure - 6: Servo regulatory response of PID controller (IMC)

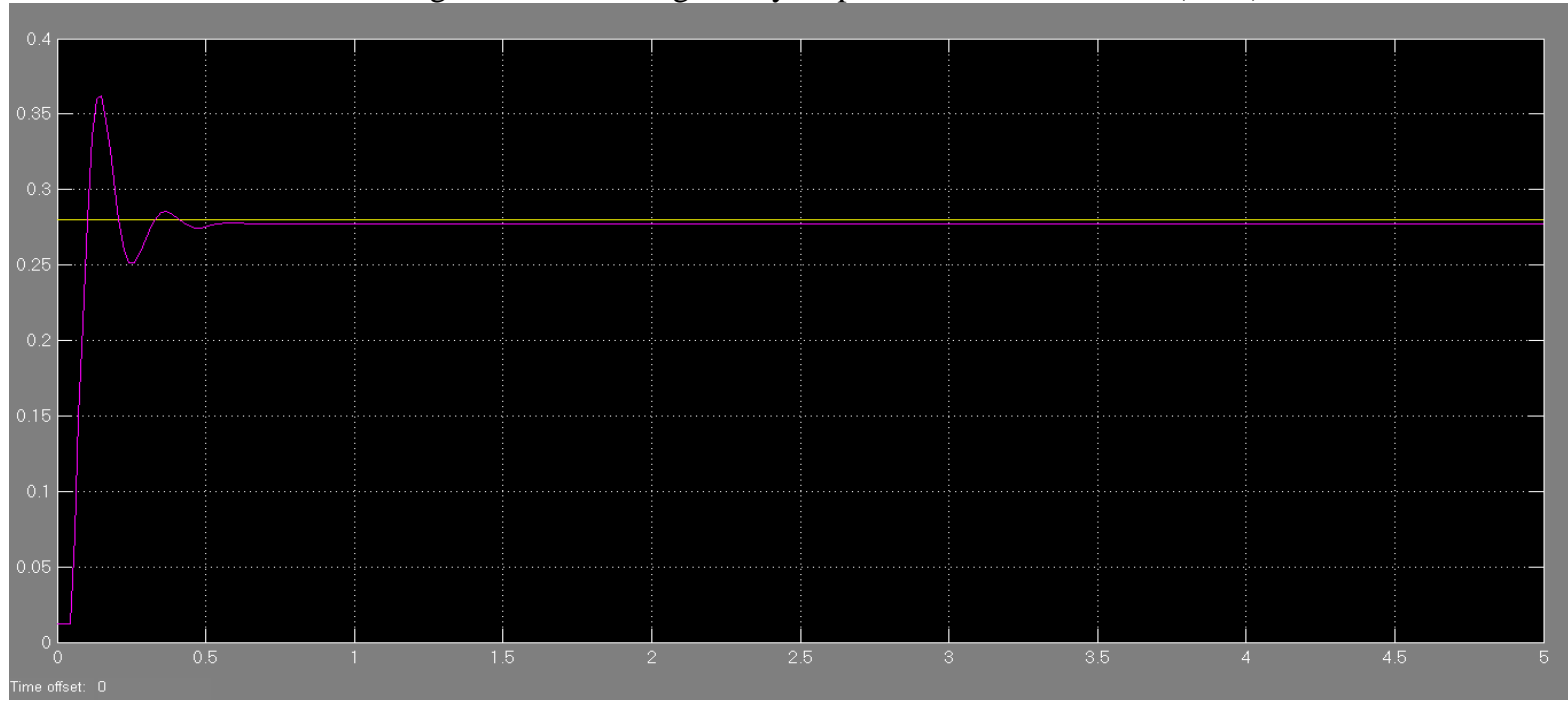

Figure - 7: Servo regulatory response of PID controller (Cohen-Coon)

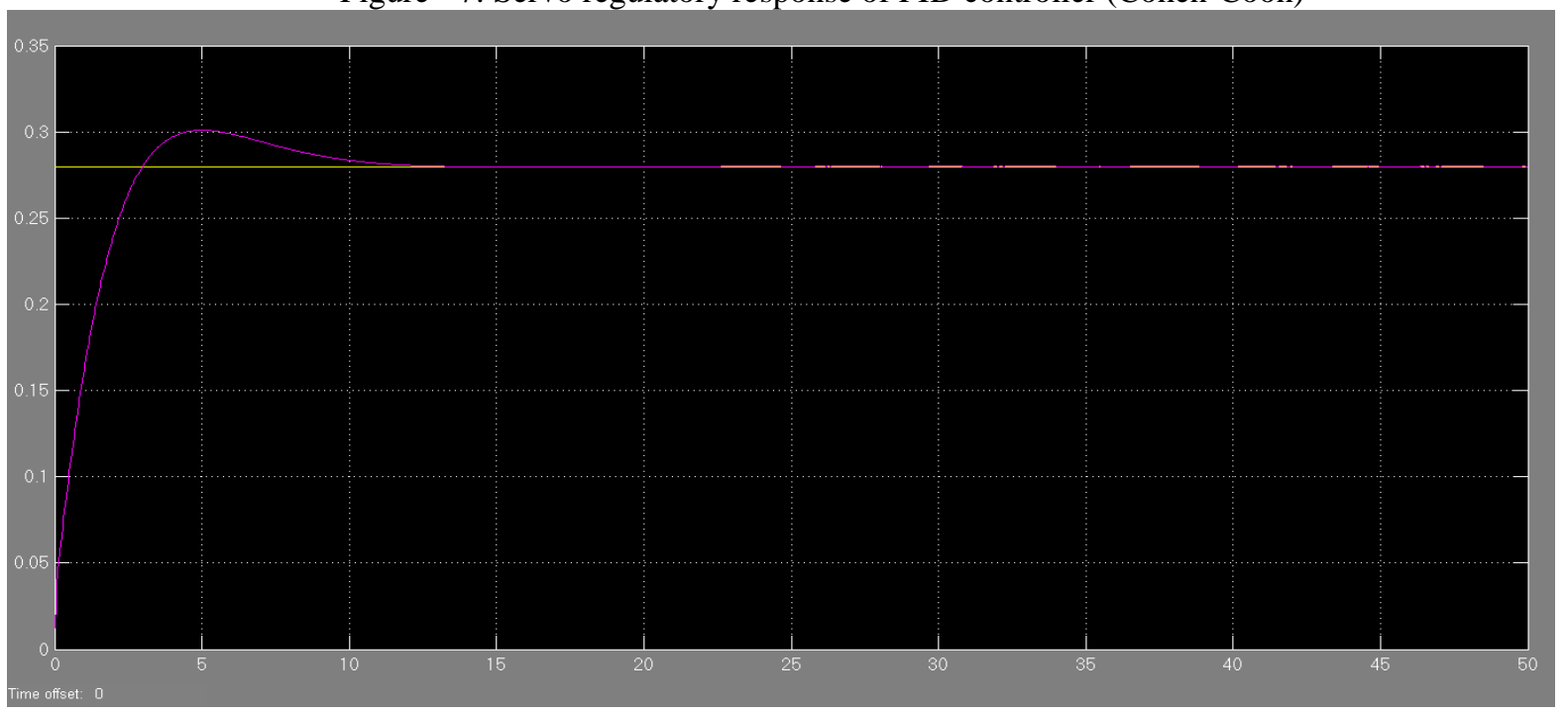

Figure - 8: Servo regulatory response of PID controller $(\mathrm{ZN})$

\section{Results And Discussion}

The various tuning techniques is designed and applied to the real time system. For the servo and regulatory responses of PID controller the performance indices were calculated and listed in the Table -2 . We infer from 
the above table that the SIMC method of control is the best suited for a spherical tank process, when compared to the IMC and other conventional controllers.

\begin{tabular}{|l|r|r|}
\hline \multicolumn{1}{|c|}{ Controller } & \multicolumn{1}{c|}{ IAE } & \multicolumn{1}{c|}{ ISE } \\
\hline Cohen Coon & 0.0816 & 0.051 \\
\hline ZN & 0.3705 & 0.0424 \\
\hline IMC & 2.803 & 0.786 \\
\hline SIMC & 0.1784 & 0.0225 \\
\hline
\end{tabular}

Table - 2: Performance indices comparison

\section{Conclusion}

For a non-linear processes IMC based PID controller settings is obtained. Its performance is tested in real time spherical tank. Comparison with SIMC and various controller tuning methods indicates the effectiveness of the proposed method. Experimental results prove that the response smooth for both servo and regulatory changes. SIMC based PI controller shows better response than other tuning methods. This is validated by IAE and ISE values. It is concluded that the SIMC based pi controller outperforms well when compared to conventional controller tuning techniques in real time. By comparing the performance indices like the IAE and ISE values of different methods, we find that the SIMC is the best method with lesser values of IAE and ISE, among all other methods like ZN, IMC, Cohen-Coon and Chien.

\section{References}

[1] Cheng Ching Yu, Auto tuning of PID controllers. A Relay feedback approach, Second Edition, Springer, 2006.

[2] Carls A. Smith \& Armando B.Corripio, Principles \& Practice of Automatic Process Control., John Wiley \& sons, New York,1985

[3] Donald R Coughnowr. PID Controllers: Process System Analysis \& Control. Second Edition, MC-Graw Hill, 1991.

[4] George Stephanopoulos, Chemical Process Control, Prentice Hall publication, 1983.

[5] Luyben W.L, Process Modeling, Simulation \& Control for chemical engineers, Mc-Graw Hill, 1990.

[6] O'Dwyer, A., Handbook of PI and PID Controller Tuning Rules. World Scientific Pub Co, London, 2003.

[7] Skogestad. S, Simple analytic rules for model reduction and PID controller tuning. Journal of Process Control, Vol 25, Pgno.85120,2004

[8] Skogestad\&ChrissGrimholt, The SIMC method for smooth PID controller tuning, Sep 7, 2011. 